\title{
Organics in meteorites - Solar or interstellar?
}

\author{
Conel M. O'D. Alexander ${ }^{1}$, George D. Cody ${ }^{2}$, Marilyn Fogel $^{2}$, and \\ Hikaru Yabuta ${ }^{2}$ \\ ${ }^{1}$ DTM, Carnegie Institution of Washington, \\ 5241 Broad Branch Road, Washington DC 20015, USA \\ email: alexande@dtm.ciw.edu \\ ${ }^{2} \mathrm{GL}$, Carnegie Institution of Washington, \\ 5251 Broad Branch Road, Washington DC 20015, USA \\ email: g.cody@gl.ciw.edu, m.fogel@gl.ciw.edu, hyabuta@gl.ciw.edu
}

\begin{abstract}
The insoluble organic material (IOM) in primitive meteorites is related to the organic material in interplanetary dust particles and comets, and is probably related to the refractory organic material in the diffuse interstellar medium. If the IOM is representative of refractory ISM organics, models for how and from what it formed will have to be revised.
\end{abstract}

Keywords. Comets, asteroids, ISM: dust

\section{Introduction}

Refractory organic material is a major component of dust in the interstellar medium (ISM), of comets, of interplanetary dust particles (IDPs) that may come from comets, of at least the surfaces of other outer Solar System bodies and of primitive meteorites (chondrites) derived from the asteroid belt. There are numerous potential mechanisms for making refractory organic material. Here we explore whether the refractory materials in chondrites, IDPs and comets are related, and whether they formed in the ISM or in the early Solar System.

\section{Relationship between IOM in meteorites, IDPs and comets}

Chondritic porous (CP-) IDPs are generally thought to be the most primitive Solar System objects available for study in the laboratory - it has been argued that they are from comets (Joswiak et al. 2000). Similarly, based on large D and ${ }^{15} \mathrm{~N}$ excesses, particularly in so-called hotspots (micron to sub-micron regions), the organic material in CP- IDPs has long been considered the most primitive available to us. An apparently macromolecular insoluble organic matter (IOM) makes up the bulk of the organic matter in chondrites (up to $\sim 2 \mathrm{wt} . \% \mathrm{C}$ ). The IOM in the most primitive chondrites is enriched in $\mathrm{D}$ and ${ }^{15} \mathrm{~N}$ in bulk (Alexander et al. 2007) and contains hotspots with similar isotopic enrichments and sizes to those in IDPs (Busemann et al. 2006). The resolvable hotspots $(>100 \mathrm{~nm}, \sim 1 \mathrm{vol} \%)$ are not sufficiently abundant to contribute significantly to the bulk compositions of the IOM. Some, but not all, hotspots are associated with roughly spherical grains (globules) that are often hollow (Garvie \& Buseck 2004, NakamuraMessenger et al. 2006). The bulk $\mathrm{H}$ and $\mathrm{N}$ isotopic compositions of chondritic IOM with the largest isotopic enrichments are comparable to the average compositions of the most primitive IDPs.

There seems to be a genetic link between the IOM in at least some chondrites and IDPs that may come from comets. Indeed, there is a striking resemblance between the 
bulk elemental compositions of comet Halley CHON particles, $\mathrm{C}_{100} \mathrm{H}_{80} \mathrm{~N}_{4} \mathrm{O}_{20} \mathrm{~S}_{2}$ (Kissel \& Krueger 1987), and the meteoritic IOM with the most anomalous isotopic compositions $\left(\mathrm{C}_{100} \mathrm{H}_{70-79} \mathrm{~N}_{3-4} \mathrm{O}_{11-21} \mathrm{~S}_{1-5}\right)$. Organic particles from Comet $81 \mathrm{P} /$ Wild 2 returned by the Stardust mission have higher $\mathrm{N}$ and $\mathrm{O}$ contents, as well as more modest $\mathrm{D}$ and ${ }^{15} \mathrm{~N}$ enrichments, compared to IOM in IDPs and primitive chondrites (Cody et al. 2008, Sandford et al. 2006). Questions still remain about how much modification (possibly even synthesis) of organics there was during capture, as well as potential sources of contamination. Perhaps more importantly, the silicate mineralogies of Wild 2 particles are not as primitive as that found in IDPs. It seems that the silicates experienced high temperatures, probably prior to formation of the Wild 2 parent body (Ishii et al. 2008). The lower abundance of presolar grains in Stardust particles also suggests that the Wild 2 material is not as primitive as IDPs or even the most primitive chondrites. If correct, it is unlikely that the IOM in Wild 2 is as primitive as that in IDPs.

\section{A Common Source?}

There is a link between the IOM in at least some primitive meteorites, IDPs and comets. Thus, this IOM must have been widespread in the early Solar System. It has been argued for some time that, because of the large $\mathrm{D}$ and ${ }^{15} \mathrm{~N}$ enrichments, some or all of the IOM must have formed in the ISM. This is supported by the similarity in the 3$4 \mu \mathrm{m}$ C-H stretch region between chondritic IOM and dust in the diffuse ISM (Pendleton \& Allamandola 2002). However, conditions would not have been so very different in the ISM and parts of the solar protoplanetary disk (nebula). Indeed, recently it has been argued that the D enrichments are solar in origin (Gourier et al. 2008).

Gourier et al. (2008) report an inverse correlation between the D-enrichment of certain functional groups and their $\mathrm{C}-\mathrm{H}$ bond energies. They propose that this correlation results from the interaction at the surface of the protoplanetary disk of $\mathrm{H}_{2} \mathrm{D}^{+}$with organic grains formed near the midplane. Alexander et al. (2007) have questioned whether there is a dependence of D-enrichment with functional group. In addition, $\mathrm{H}_{2} \mathrm{D}^{+}$is so reactive that it should exchange more-or-less indiscriminately, rather than as a function of C$\mathrm{H}$ bond energy, which seems to be borne out by recent experiments (Thomen et al. 2008).

While the Gourier et al. model remains controversial, they raise an important issue, namely: how does one distinguish between organic material formed in the ISM and solar nebula? To try to address this issue, we have analyzed the IOM in a wide range of primitive meteorites using a number of techniques (Alexander et al. 2007, Cody \& Alexander 2005). We have found considerable elemental and isotopic variation in IOM composition between meteorites. If the IOM in all meteorites has an interstellar origin, these variations must be consistent with evolution from a single precursor material. On the other hand, if the IOM is solar, one could expect to see systematic variations in composition and abundance as conditions changed over the 2-3 Ma that different chondrite groups seem to have formed.

In the most primitive chondrites, the ratio of IOM to presolar nanodiamonds is a fairly constant, as are the abundances of both in the low temperature matrices of chondrites (Alexander 2005, Huss \& Lewis 1995). The IOM in all chondrites also seems to be associated with trapped noble gases that may be interstellar (Huss et al. 1996). These observations are consistent with a common, probably interstellar, origin for IOM. However, even in the most primitive chondrites there is a considerable range in IOM compositions, and the abundance ratios of IOM to presolar grains other than nanodiamonds are more variable. 
Much of this variation relative to other presolar grains could simply reflect alteration on the meteorite parent bodies. For instance, presolar silicates are easily destroyed during aqueous alteration and thermal metamorphism. The IOM will also be sensitive to these processes. What are generally considered the most primitive chondrites (CI, CM, CR and Tagish Lake) have all experienced aqueous alteration. The IOM in these meteorites exhibits a wide range of aliphatic/aromatic $\mathrm{C}$ ratios $(\mathrm{CR}>\mathrm{CI}>\mathrm{CM}>$ Tagish Lake) that appears to be the result of variable oxidation of the aliphatic fraction of a common precursor at the onset of alteration (Cody \& Alexander 2005). In general, the bulk IOM $\mathrm{D}$ enrichments decline in the same order as the aliphatic/aromatic ratio. However, there are some notable exceptions. The most dramatic is to be found in the CMs. All CMs have IOM with very similar elemental and functional group chemistries, and most have quite uniform isotopic compositions $\left(\delta \mathrm{D}=800 \%\right.$ o, $\delta^{13} \mathrm{C}=-18 \%$, and $\delta^{15} \mathrm{~N}=0 \%$ ). However, the IOM in the CM Bells $\left(\delta \mathrm{D} \approx 3000 \%\right.$ o, $\delta^{13} \mathrm{C} \approx-34 \%$, and $\delta^{15} \mathrm{~N} \approx 400 \%$ o has a composition that more closely resembles that of the IOM in the CRs, the chondrite group with the generally most isotopically anomalous material. Bells experienced a somewhat different mode of alteration than the other CMs, suggesting that the degree to which IOM is modified during parent body processing can be very sensitive to the conditions.

This sensitivity to conditions can also be seen in those meteorites that experienced thermal metamorphism (CV, CO, O and E chondrites). The metamorphosed chondrites all have lower $\mathrm{H} / \mathrm{C}, \mathrm{O} / \mathrm{C}$ and $\mathrm{N} / \mathrm{C}$ ratios than the aqueously altered ones. This is to be expected as thermal maturation is well known to drive the evolution of carbonaceous materials towards more graphite-like structures, with the associated loss of heteroatoms. The trends in $\mathrm{H} / \mathrm{C}$ vs. $\mathrm{N} / \mathrm{C}$ and $\mathrm{O} / \mathrm{C}$ are all consistent with evolution from a CR/CM-like precursor. Indeed, CMs that have been shock heated roughly parallel the trend in the metamorphosed chondrites, even though the IOM in most metamorphosed chondrites experienced destructive oxidation in addition to thermal maturation. More perplexing are the isotopes. The expectation would be that the $\mathrm{D}$ enrichment would decrease with increasing degree of metamorphism. Except in the $\mathrm{O}$ chondrites, this is what is seen. In the $\mathrm{O}$ chondrites $\mathrm{D}$ enrichments increase dramatically with increasing metamorphism. At present, there is no good explanation for this behavior.

The variation in elemental and functional group chemistry of IOM within and between chondrite groups is consistent with parent body modification of a common precursor that looked something like the IOM in the CR chondrites. The isotopes paint a more complicated story that we do not fully understand yet, but it is clear that conditions during parent body processing played a central role. It is not yet possible to see through the parent body processing to the original material in all chondrite groups. However, if one uses the IOM from the most primitive members of each chondrite group, there is no apparent correlation between either IOM composition or its abundance in the matrix and crude estimates for the time and conditions of formation of the chondrite parent bodies.

\section{A Solar or ISM Origin?}

Based on current evidence, it seems likely that there was a widespread IOM-like precursor material in the formation regions of asteroids and at least some comets. Whether this formed in the ISM or Solar System remains uncertain, although the relatively constant presolar nanodiamond/IOM ratios in chondrites, along with the close similarities in the IR 3-4 $\mu \mathrm{m}$ C-H stretch region between the IOM and diffuse ISM, point towards an interstellar origin. Refractory organic material is abundant in the ISM, and to date it has not been shown that IOM-like material can be made in the solar nebula on the scale required. Nevertheless, the IOM differs in significant ways from what has been 
inferred for refractory organics in the diffuse ISM. The diffuse ISM material is thought to be composed of large PAHs derived from C-rich AGB stars (Kwok 2004, Pendleton \& Allamandola 2002). One way we are pursuing of reconciling this model with an IOM that is dominated by small, highly substituted PAHs is if the large PAHs are heavily damaged by cosmic rays while trapped in ices in molecular clouds. The damaged regions would be hydrogenated, nitrogenated and oxygenated (e.g., Bernstein et al. 2003), leaving only small islands of highly substituted PAHs surrounded by short, highly branched aliphatic material. These damaged regions would take on the $\mathrm{H}, \mathrm{N}$ and $\mathrm{O}$ isotopic compositions of the ice, which would be rich in D and ${ }^{15} \mathrm{~N}$. If returned to the diffuse ISM, this process would somehow have to be reversed, but material that was incorporated into the Solar System directly from the protosolar molecular cloud would not have experienced this 'annealing' process.

The $\mathrm{C}$ and $\mathrm{N}$ isotopes remain problematic to this appealing picture. The $\mathrm{C}$ and $\mathrm{N}$ isotopes of PAHs formed around C-stars should be far from the solar composition judging from models, astronomical observations and presolar grains. However, the PAHs in IOM are on average essentially solar in their C isotopic compositions (Sephton et al. 2000). It cannot be ruled out that on average $\mathrm{PAH}$-producing stars have solar $\mathrm{C}$ isotopic compositions, but this seems unlikely at present. The $\mathrm{N}$ isotopic compositions of AGB stars are always highly depleted in ${ }^{15} \mathrm{~N}$ compared to solar, whereas the bulk and subgrain ${ }^{15} \mathrm{~N}$ abundances in the most primitive IOM are enriched. Hence, if the IOM is interstellar in origin and is representative of the refractory organic dust in the ISM, how and from what it formed will have to be rethought.

\section{Acknowledgements}

We gratefully acknowledge financial support from NASA's Origins of Solar Systems and Astrobiology programs.

\section{References}

Alexander, C. M. O'D. 2005, Meteorit. Planet. Sci., 40, 943

Alexander, C. M. O'D., Fogel, M., Yabuta, H., \& Cody, G. D. 2007, Geochim. Cosmochim. Acta, 71,4380

Bernstein, M. P., Moore, M. H., Elsila, J. E., Sandford, S. A., Allamandola, L. J., \& Zare, R. N. 2003, ApJ (Letters), 582, L25

Busemann, H., Young, A. F., Alexander, C. M. O'D., Hoppe, P., Mukhopadhyay, S., \& Nittler, L. R. 2006, Science, 314, 727

Cody, G. D. \& Alexander, C. M. O'D. 2005, Geochim. Cosmochim. Acta, 69, 1085

Cody, G. D., et al. 2008, Meteorit. Planet. Sci., 43, 353

Garvie, L. A. J. \& Buseck, P. R. 2004, Earth Planet. Sci. Lett., 224, 431

Gourier, D., Robert, F., Delpoux, O., Binet, L., Vezin, H., Moissette, A., \& Derenne, S. 2008, Geochim. Cosmochim. Acta, 72, 1914

Huss, G. R. \& Lewis, R. S. 1995, Geochim. Cosmochim. Acta, 59, 115

Huss, G. R., Lewis, R. S., \& Hemkin, S. 1996, Geochim. Cosmochim. Acta, 60, 3311

Ishii, H. A., et al. 2008, Science, 319, 447

Joswiak, D. J., Brownlee, D. E., Pepin, R. O., \& Schlutter, D. J. 2000, Lunar Planet. Sci., 31, \#1500 (abs.)

Kissel, J. \& Krueger, F. R. 1987, Nature, 326, 755

Kwok, S. 2004, Nature, 430, 985

Nakamura-Messenger, K., Messenger, S., Keller, L. P., Clemett, S. J., \& Zolensky, M. E. 2006, Science, 314, 1439

Pendleton, Y. J. \& Allamandola, L. J. 2002, ApJS, 138, 75

Sandford, S. A., et al. 2006, Science, 314, 1720 
Sephton, M. A., Pillinger, C. T., \& Gilmore, I. 2000, Geochim. Cosmochim. Acta, 64, 321

Thomen, A., Robert, F., \& Derenne, S. 2008, Lunar Planet. Sci., 39, \#2001 (abs.)

\section{Discussion}

Cruikshank: Conel, you made a point noting that the bulk of the organic materials is not FTT synthesized. I guess in a large part because it's not associated with mineral grains. Yet in George Flynn's talk, he spoke of an organic rim on mineral grains that may not be the bulk of the organic material in the IDP. But is it possible to distinguish by experimental or other means the difference in the nature of organic material on a mineral grain which may have been FTT synthesized compared to the other material?

Alexander: People have looked at this to some extent. You do see in meteorites and in IDPs carbonaceous material rimming some grains. In the meteorites it's certainly not as pervasive as George Flynn implied. Generally that material, in the TEM at least, looks more graphitic. I don't know if this is true in the IDPs, but certainly in the meteorites the stuff that is rimming the minerals looks more like poorly graphitized carbon, i.e. it exhibits d-spacings, whereas the stuff you see that is unassociated with minerals is essentially featureless.

SANDFORD: You look at all these residues from many, many meteorites and you have done this by using solvents, so presumably you are not necessarily seeing the primordial $\mathrm{D}$ to $\mathrm{H}$ ratio from anything that is exchangeable. So do you have a sense of what the values that would have been in the original meteorite but were exchanged during the dissolution process.

AlEXANDER: When we've looked at meteorites in situ, generally we see the same range of compositions of hot-spots. But the trouble when you are looking at the meteorites is that much of the hydrogen is in hydrous phases. So it's hard to make a really good comparison. People have done experiments back in the ' $80 \mathrm{~s}$ using labeled compounds, e. g., labeled acids, labeled solvents, etc. There's some disagreement, but generally the feeling is that you don't have more than a few percent exchange of hydrogen. You always have to worry about that, but for most purposes I think we don't get a great deal of exchange. All our isolations were done at room temperature, whereas in the old days you would heat things in acids at $60-80{ }^{\circ} \mathrm{C}$.

SANDFORD: In a way, that's a big clue telling you a lot of hydrogen is not in exchangeable forms.

Alexander: Well, that's not entirely true. It is exchangeable. Hikaru Yabuta did hydrothermal experiments and showed that if you heat these things for a couple of days at $300{ }^{\circ} \mathrm{C}$ under modest pressure then you entirely exchanged the hydrogen. We don't know what the kinetics of that the process is, and that is something we need to work out.

Kwok: When you say large PAHs, do you mean pure aromatic material or a mixture of aromatic and aliphatic materials, e. g., the kerogen-like compounds that we are seeing in protoplanetary nebulae and planetary nebulae?

AlEXANDER: What I would say is that we have this macromolecular material that seems to be composed of aliphatic material binding together small PAHs. We don't think there's more than a few percent of PAHs with larger than 3 to 4 rings. This material is a 
macromolecular material with about $40-60 \%$ of the carbon in aromatic form, but that material is in small PAHs, not in coronene or larger PAHs.

ZiURYs: Maybe these small PAH aromatic rings are responsible for what we see in the interstellar medium, not the big ones.

Alexander: That's quite possible.

SLOAN: Maybe I missed this, but why do you say the PAHs aren't from the AGB stars?

AlExAnDER: The carbon isotopes of the organic materials are essentially solar, whereas the carbon isotope ratios in AGB star atmospheres and in presolar grains in meteorites from AGB stars are far from solar. Unless you have a magic way of mixing all these material up to make solar composition, there can't be a large fraction of AGB material ending up in the IOM.

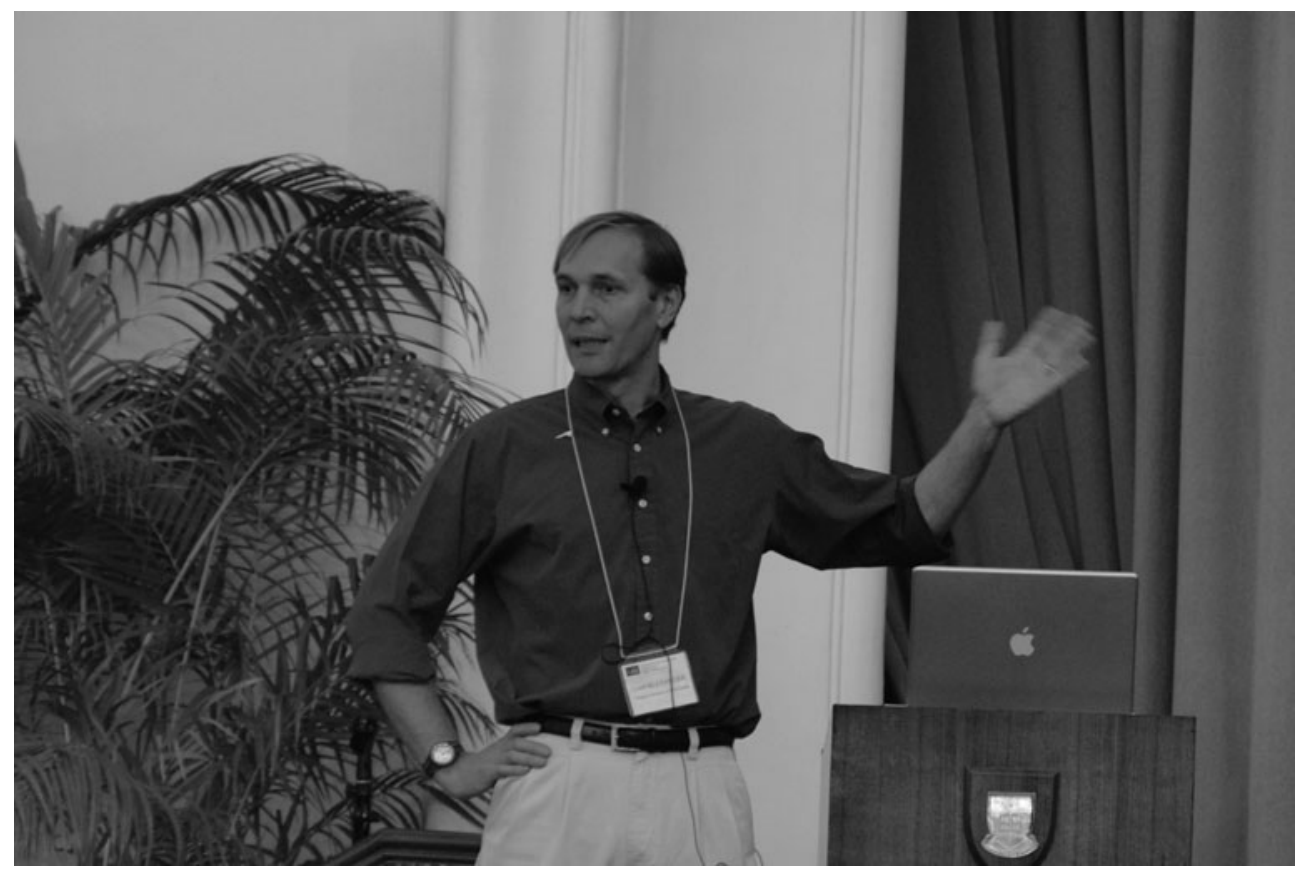

Conel Alexander (photo by Sze-Leung Cheung). 